\title{
Vertauschte Kinder: Krankenhaus muss zahlen
}

\author{
Krankenanstalten werden im Bereich der Neonatologie vom Gesetz \\ besonders streng in die Pflicht genommen. Der OGH urteilte über die \\ Vertauschung eines Kindes, die vor 20 Jahren passiert ist. \\ Von Monika Ploier
}

Ein Ehepaar findet nach über 20 Jahren heraus, dass seine Tochter - infolge einer Verwechslung als Neugeborene auf der Geburtenstation - nicht sein leibliches Kind ist. Im Zuge des gerichtlich durchgeführten Beweisverfahrens zeigte sich, dass das Kind mittels Kaiserschnitt entbunden wurde und der Mutter ca. 2 Stun- renen jedenfalls einen gravierenden Fall von Organisationsverschulden darstelle und diese als „grobe Fahrlässigkeit“ zu bewerten sei. Aufgrund des Behandlungsvertrages schuldet eine Geburtenstation alle organisatorischen Vorkehrungen, um Vertauschungen zu verhindern und die Einhaltung der Anweisungen auch zu kontrollieren. Anders als bei anderen Behandlungsverträgen ist hinsichtlich der korrekten $\mathrm{Zu}$ ordnung und Übergabe an die Obsorgeberechtigten jedenfalls ein konkreter Erfolg geschuldet. Grundsätzlich erfordert ein grob fahrlässiges Organisa-

den nach der Geburt, als sie von der Vollnarkose noch benommen war, mitgeteilt wurde, dass sie eine Tochter bekommen hatte. Der Vater, der während der Entbindung nicht anwesend war, nahm das Kind einige Stunden später von der Frühgeburtenstation mit zur Mutter in deren Krankenzimmer. Dies war die erste bewusste Begegnung von Mutter und Tochter. Im Beweisverfahren zeigte sich, dass im Krankenhaus eine Vorgabe bestanden hatte, wonach Neugeborene nicht aus der Frühgeburtenstation gebracht werden durften. Das Pflegepersonal ließ dies jedoch ohne die vorgeschriebene Abbefundung durch den Kinderarzt zu. Damit stand fest, dass ein Vertauschen des Kindes nicht nach dem erstmaligen Kontakt stattgefunden haben konnte, sondern vielmehr unmittelbar nach der Geburt passiert sein musste. Wodurch es zu der Verwechslung gekommen ist, konnte nicht mehr festgestellt werden.

Es zeigte sich, dass bei einer Kaiserschnittentbindung die zuständige Hebamme das neugeborene Baby aus dem Operationssaal in das Erstversorgungszimmer bringen muss, wo es vom Kinderarzt übernommen und erstversorgt wird. Im gegenständlichen Fall war ein Namensband mit dem Namen der Mutter und deren Geburtsdatum vorbereitet, das nach der Übergabe mit Tag und Stunde der Geburt sowie Geschlecht des Kindes sowie dem Namen der Hebamme vervollständigt wurde. Frühgeborene werden auf die Frühgeburtenstation - einen größeren Raum, in dem bis zu zehn Kinder Platz finden - gebracht. Dort findet üblicherweise jeder Säugling ein eigenes beschriftetes Bett vor, in das er stets gelegt wurde. Zutritt hatten in unserem Fall nur die Eltern der Neugeborenen.

Sowohl das Erstgericht als auch in weiterer Folge der OGH haben ausgesprochen, dass diese Vertauschung eines Neugebo- tionsverschulden einen objektiv und auch subjektiv schweren Verstoß gegen die Anforderungen der im Verkehr erforderlichen Sorgfalt. Gerade die Sorgfaltsanforderungen an eine Geburtenstation sind besonders hoch, da sowohl Persönlichkeitsrechte aller Beteiligten betroffen sind (Familie, Identität, biologische Abstammung) als auch die Schutzbedürftigkeit der Betroffenen. Eine werdende Mutter muss den Abläufen im Kreißsaal und der geordneten Organisation auf der Neonatologie vertrauen dürfen. Um dieses Vertrauen zu schützen, sei die Verpflichtung der Krankenanstalt äußerst streng zu sehen. Sie muss geeignete Vorkehrungen gegen derartige Kindsvertauschungen treffen.

Die Eltern werden aufgrund der Kindesvertauschung nie erfahren, was mit ihrem leiblichen Kind passiert ist. Das betroffene Mädchen hat über 20 Jahre in dem Glauben gelebt, bei seinen leiblichen Eltern aufzuwachsen, um dann zu erfahren, dass seine Identität auf einem Irrtum beruht. Die biologische Herkunft bleibt für immer verborgen. Daher bejahten die Gerichte einen Anspruch auf Trauerschmerzengeld. //

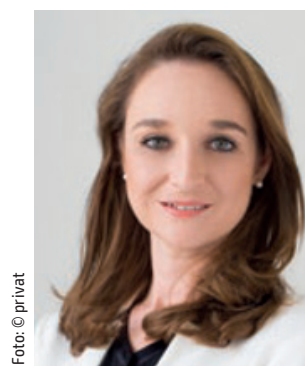

Dr. MONIKA PLOIER ist Anwältin bei HLMK Rechtsanwälte und auf Medizinund Arbeitsrecht spezialisiert. Sie ist Verfasserin zahlreicher Publikationen und Lektorin für Medizin \& Recht an mehreren akademischen Bildungseinrichtungen. Monika Ploier ist Obfrau des Forschungsinstituts für Recht in der Medizin FIRM. 